\title{
LCOS Spatial Light Modulator for Digital Holography
}

\author{
Weijie Wu, Mike Pivnenko, Daping Chu* \\ Center for Photonics Devices and Sensors, University of Cambridge, Cambridge CB3 OFA, UK
}

Received November 14, 2021; accepted December 06, 2021; published December 31, 2021

\begin{abstract}
Liquid crystal on silicon (LCOS) spatial light modulator (SLM) is the most widely used optical engine for digital holography. This paper aims to provide an overview of the applications of phase-only LCOS in two-dimensional (2D) holography. It begins with a brief introduction to the holography theory along with its development trajectory, followed by the fundamental operating principle of phase-only LCOS SLMs. Hardware performance of LCOS SLMs (in terms of frame rate, phase linearity and flicker) and related experimental results are presented. Finally, potential improvements and applications are discussed for futuristic holographic displays.
\end{abstract}

The concept and method of holography were proposed by Polish scientist Mieczysław Wolfke in 1920 when he studied the possibilities of optical imaging of molecular structures [1], which were later independently discovered and developed by Dennis Gabor [2]. However, the real holographic display failed to implement due to the lack of strong and coherent light sources until lasers emerged in the 1960s [3]. Holographic display, named as a full parallax display technology in Benton's framework [4], can provide all four physical depth cues that the human brain can perceive (i.e. binocular parallax, motion parallax, accommodation, and convergence) [5]. It is eulogized as the 'holy grail' for three-dimensional (3D) displays due to its capability to deliver true 3D images for naked eyes.

The original holography utilizes the interference principle to record both amplitude and phase information of a 3D object on photographic films [6]. However, it was shown phase-field of holography functions could also be used to represent the spatial and optical properties of an object, with all depth cues, thus provide a vivid 3D sensation [8].

In 1967, Lohmann and Paris calculated a hologram by the computing power of a digital computer [9]. Following Goodman and Lawrence's idea of digital holography and Yaroslavskii and Merzlyakov's fundamental theory, the technique of computer-generated hologram (CGH) arose [10]. A CGH is a digital form of a suitable hologram pattern, calculated (typically based on Fourier transform) to represent the amplitude and phase information of the desired object, which supersedes the optical information recording process mentioned above. The $\mathrm{CGH}$, which loads on an optical engine like a SLM in the form of 2D pixel arrays, is independent of the holographic recording material. Furthermore, the CGH calculation process can reproduce all different functions of diffractive optical

\footnotetext{
*E-mail: dpc31@cam.ac.uk
}

elements (ODE) and even manifest the information of non-existent objects, making it enormously useful for data manipulation, transmission, and replication. Due to this numerical-based feature, both amplitude and phase information can be separately extracted as amplitude-only and phase-only, respectively. As human eyes are incentive to phases, it is possible to use phase-only information in the form of phase-only $\mathrm{CGH}$ to reconstruct not only the positioning information but also the intensity of an object. Such phase-only CGHs can be uploaded as the information source on phase-only SLMs which are universally used in holographic displays during the object's image retrieve process. Various algorithms for CGH calculations have been developed over the years, from the primitive direct binary search (DBS), simulated annealing (SA) [11], Gerchberg -Saxton (G-S) [12], to other complex ones.

The first liquid crystal pixel array driving by silicon backplane was reported by the team of Ernstoff et al. in the early 1970s [13]. Shortly afterward, the first LCOS demo was shown by Crossland et al. in 1982 based on the field-effect of nematic liquid crystal [14]. Over the last forty years, LCOS devices have been intensively studied as a technology for SLMs in multifarious applications, such as holographic display, optical correlators [15], optical tweezers [16], and wavelength selective switches [17]. LCOS SLM acts as the optical engine in digital holography for both hosting the $\mathrm{CGH}$ and modulating the reference beam for image reconstruction. A phase-only LCOS SLM consists of two alignment layers, one liquid crystal (LC) layer for phase-modulating, and one electrode layer for signal-addressing $[18,19]$. The electrode layer is a pixel array, where the voltage of each pixel can be individually controlled according to the input signal, i.e. CGH. Due to the coordination of the electrode pixel voltage and local LC molecule rotation, the phase delay provides the optical information required at that pixel. For a reflective LCOS SLM, the pixelated electrode layer is made of aluminium mirrors; while for a transmissive LCOS SLM, the pixelated electrode layer is made of transparent conductors. Complementary metal-oxide-semiconductor (CMOS) silicon backplanes are used for reflective LCOS SLMs, which according to their memory accessing properties are either DRAM-based or more often SRAM-based [20].

The nematic LC is prevalently adopted in phase-only 
applications due to its large birefringence and multilevel phase modulation ability [18, 19, 21-24]. When designing a suitable LC layer for LCOS for display applications, it is preferred to use the LC cell with the minimum thickness, larger splay elastic constant, and the smallest dielectric constant ratio on the premise of a $2 \pi$ modulation depth and a proper LC response speed [25].

A disadvantage of using nematic LCs in phase-only LCOS SLMs is from its anti-parallel zero-twisted configuration. When the applied voltage is removed, apart from the slowdown of the LC molecules rotating back to their original state due to the viscous dragging force, the relaxation of the LC molecules within the mid-layer will be forced to the opposite direction because of the unwanted asymmetric backflows of the LC molecules within the two adjacent LC layers, which will further delay the optical response of the whole device [18]. As a result, the intrinsic LC relaxation time, which is the off-time when the applied voltage is removed, is several times longer than the on-time when a voltage is applied, resulting in a long frame time for the breakups in colour sequential operations [26] and image blurring during refreshing [28] as shown in Figure 1. A thinner LC layer thickness would moderate this issue [18]. However, to maintain a $2 \pi$ modulation depth in a thin LC layer requires LC materials of high birefringence. An attempt for high birefringence mixture showed material instability at high temperatures and photochemical degradation under blue light exposure [27]. Alternatively, high frame rates can be achieved by using nematic LCs with moderately high birefringence and low visco-elastic ratio in combination with a voltage adjustment method and LCOS SLMs with a refresh rate higher than 2,000 frames per second (fps) were reported [28].
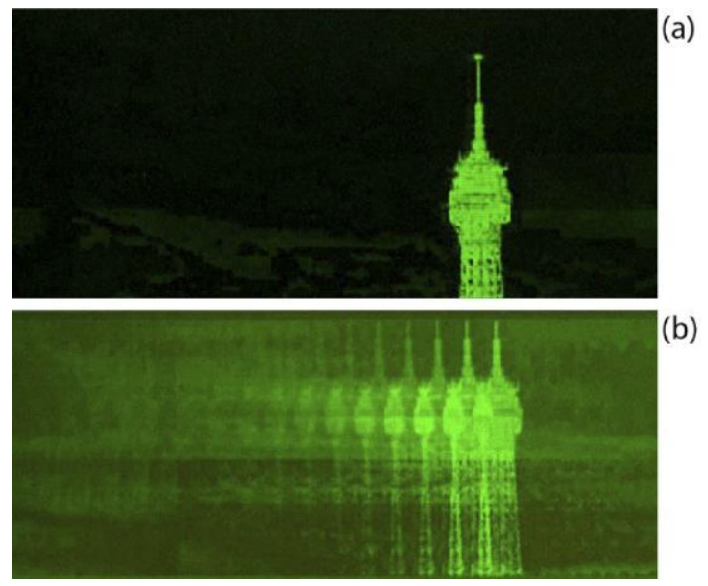

Fig. 1. Blurring effect of a refreshing image on (a) a fast LCOS with a response time of $\sim 700 \mu$ s and (b) a slow LCOS device with a response time of $\sim 30 \mathrm{~ms}[28]$.

Nematic LCs are known to be electro-optically nonlinear, and AC driving schemes, especially digital pulse width modulation (PWM) driving schemes, can make the LC molecule directions and hence the phase delays wobbling in a given phase level, which is known as phase flicker [29]. Such an instability is due to the competition between the corresponding LC molecules' relaxation and the change in their electrical driving force, leading to temporal rotational fluctuations of the forced LC molecules and modulated phase instability. Figure 2 shows an example of phase flicker induced by a digital PWM driving waveform.

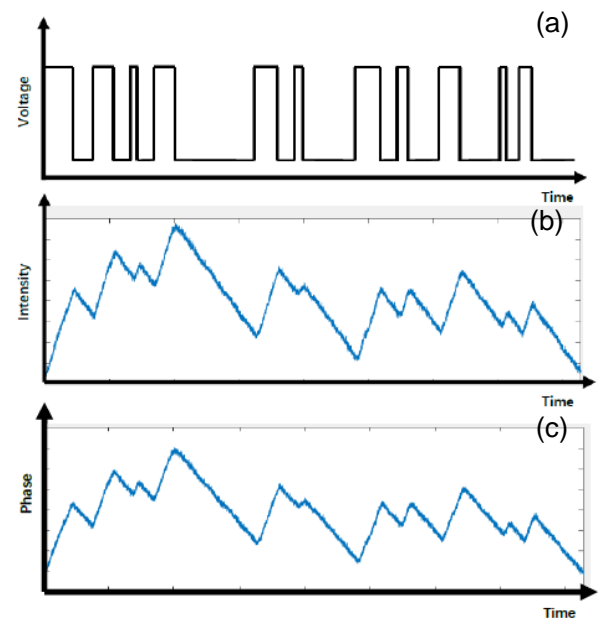

Fig. 2. (a) The PWM driving waveform, (b) the corresponding instant intensity, and (c) the instant phase calculated at grey level 120 within a single modulation period.

Although in a continuous display, the effect of phase flicker is less obvious as the flicker frequency is normally higher than that can be perceived by human eyes [30]. However, in instant holographic reconstruction or other non-display applications, phase flicker is the culprit of instantaneous errors, especially crosstalk. Furthermore, phase flicker can also lead to phase overlapping between adjacent phase levels, limiting the number of the phase levels and hence the information bandwidth that an LCOS SLM can deliver.

To minimise the phase flicker, the signal pulse of each grey level should be arranged as uniformly as possible. Averaged phase flicker of $0.0018 \pi$ was reported in a $6 \mu \mathrm{m}$ pixel LCOS SLM for 8-bit infrared modulation with manually optimized driving patterns [31]. In which, the phase flicker was reduced to such a level to eliminate overlap of the adjacent phase levels for 8-bit modulation, as shown in figure 3 . As a result, a $17.7 \%$ improvement on the holographic reconstructed image in terms of RMS contrast ratio was achieved by a $50 \%$ flicker reduction together with phase linearisation, as shown in Fig. 4. This is followed by a deep learning method of pattern optimization for an averaged phase flicker of $0.0011 \pi$ and 10-bit modulation [32]. Other LCOS driving methods including high-frequency field inversion and DC balancing failed to reach the same optimization effect [33]. Finally, the phase flicker can be reduced through changing LC 
properties. A study successfully reduced the flicker up to $80 \%$ by increasing the LC's viscosity when cooling it to $-8{ }^{\circ} \mathrm{C}$, but the switching speed become much slower [34].
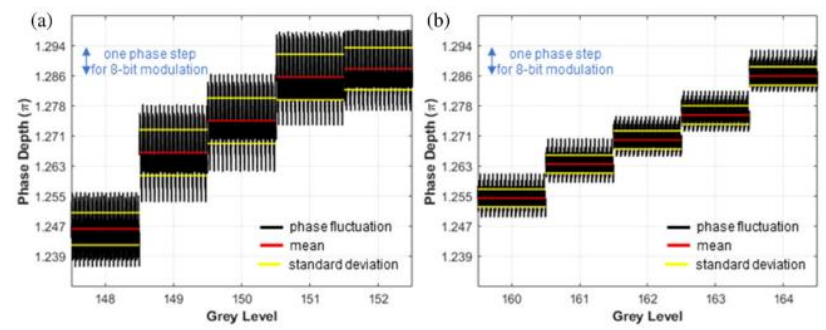

Fig. 3. Phase fluctuations at phase depth around $1.26 \pi$ (a) before and (b) after optimization on phase linearity and phase flicker [31].

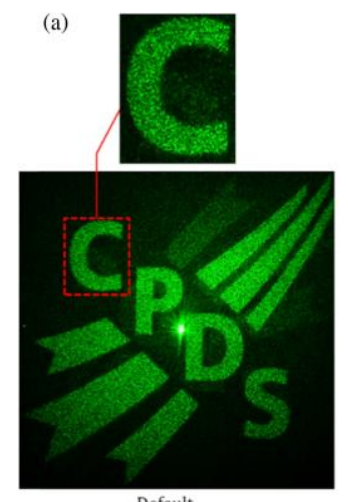

Default

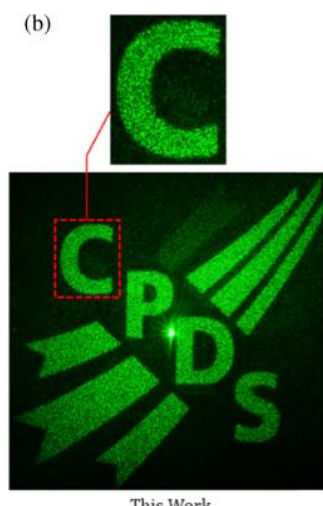

This Work
Fig. 4. Holographic reconstructed image (a) before and (b) after optimization from work [31].

In conclusions, the market of digital holography using phase-only LCOS SLMs will continue to grow. After the introduction of 'Metaverse', LCOS SLMS are bound to play a vital role for a more parallel, synchronized, and immersive end-user experience. At the same time, the requirements for LCOS SLMs will be more stringent. As high display quality, in terms of high refresh rate, high display resolution and low phase flicker, is the essential for the promised holographic world. Better driving techniques for a faster switching, smaller phase flicker and higher phase levels should be attempted. Finally, phase retrieving algorithms for $\mathrm{CGH}$ calculations should be further developed to incorporate phase flicker and other effects if possible.

\section{References}

[1] M. Wolfke, Physikalische Zeitschrift 21, 495 (1920).

[2] D. Gabor, Nature 161, 777 (1948).

[3] H. Haken, Light and Matter 5, 14 (1970).

[4] S. Benton, Selected Papers on Three-dimensional displays, SPIE Press (2001).

[5] X. Liang et al., 3D holographic display with optically addressed spatial light modulator, 3DTV-CON 2009 - 3rd 3DTV Conference (2009).

[6] J. Chen, W. Cranton, M. Fihn, Handbook of Visual Display Technology (Springer 2012).
[7] D. Rogers, The chemistry of photography: From classical to digital technologies (Royal Society of Chemistry, 2007).

[8] S. Reichelt et al., Proc. SPIE 7690, 76900B (2010).

[9] A.W. Lohmann, D. Paris, Appl. Opt. 6, 1739 (1967).

[10] J.W. Goodman, R.W. Lawrence, Appl. Phys. Lett 17, 77 (1967).

[11] D.C. O’Brien, R.J. Mears, W.A. Crossland, Appl. Opt. 33, 2795 (1994).

[12] R.W. Gerchberg, W.O. Saxton, Optik 35, 237 (1972).

[13] M. Ernstoff, A. Leupp, M. Little, and H. Peterson, Liquid crystal pictorial display, Proceedings of the 1973 International Electron Devices Meeting, IEEE, 548 (1973).

[14] W.A. Crossland, P.J. Ayliffe, P.W. Ross, Proc SID 23, 15 (1982).

[15] M. Tang, J. Wu, Optical Correlation recoginition based on LCOS, Internation Symposium on Photoelectronic Detection and Imaging 2013, Optical Storage and Display Tech., 8913 (2013).

[16] A. Hermerschmidt et al., Holographic optical tweezers with real-time hologram calculation using a phase-only modulating LCOS-based SLM at $1064 \mathrm{~nm}$, Complex Light and Optical Forces II, International Society for Optics and Photonics, 30282 (2008).

[17] M. Wang, et al. Photonics 4, 22 (2017).

[18] Z. Zhang, Z. You, and D. Chu, Light Sci. \& Appls. 3, e213 (2014).

[19] D. Yang, and S. Wu, Fundamentals of liquid crystal devices, 2nd edition (Wiley 2015).

[20] B. Prince, Semiconductor memories: A handbook of design, manufacture, and application, 2nd edition (John Wiley \& Sons 1996).

[21] J.C. Jones, Liquid crystal displays, Handbook of optoelectronics: Enabling Technologies, 2nd ed. (CRC Press 2018).

[22] A. Adrian et al., Phys. Wave Phenom. 27, 67 (2019).

[23] S.M. Kelly, M. O’Neil, Liquid crystal for electro-optic applications, Handbook of advanced electronics and photonic materials and devices (2000).

[24] Y. Ji et al., J. SID 22, 4652 (2015).

[25] X. Chang, Solution-processed $\mathrm{ZnO}$ nanoparticles for optically addressed spatial light modulator and other applications, Ph.D. thesis (University of Cambridge, Cambridge 2019).

[26] E. Moon et al., Opt. Express 22, 6526 (2014).

[27] G. Aad et al., Phys Rev. D 83, 4389 (2011).

[28] M. Pivnenko, K. Li, D. Chu, Opt. Express 29, 24614 (2021).

[29] H. Yang, D.P. Chu, Opt. Express 27, 24556 (2019).

[30] P. Bach-Y-Rita et al. Int. J. Hum. -Comput. Interact 15, 285 (2003).

[31] Y. Tong, M. Pivnenko, D. Chu, Appl. Opt. 58, G248 (2019).

[32] Y. Tong, M. Pivnenko, D. Chu, Adv. Dev. \& Instr. 1, 10 (2020).

[33] H. Yang, D. Chu, Opt. Express 27, 24556 (2019).

[34] J. García-Márquez, et al. Opt.Express 16, 8431 (2008). 